Web Semant. 2006 ; 4(3): 181-195. doi:10.1016/j.websem.2006.05.007.

\title{
The foundational model of anatomy in OWL: Experience and perspectives
}

\author{
Christine Golbreich ${ }^{\mathrm{a}}$, Songmao Zhang ${ }^{\mathrm{b}}$, and Olivier Bodenreider ${ }^{\mathrm{C}}$ \\ Christine Golbreich: Christine.Golbreich@univ-rennes1.fr; Songmao Zhang: smzhang@math.ac.cn; Olivier Bodenreider: \\ olivier@nlm.nih.gov \\ a LIM, University Rennes 1, 35043 Rennes, France \\ ${ }^{b}$ Academy of Mathematics and System Sciences, Chinese Academy of Sciences, Beijing 100080, \\ PR China \\ c U.S. National Library of Medicine, 8600 Rockville Pike MS 43, Bethesda, MD 20894, USA
}

\begin{abstract}
We present the method developed for migrating the Foundational Model of Anatomy (FMA) from its representation with frames in Protégé to its logical representation in OWL and our experience in reasoning with it. Despite the extensive use of metaclasses in Protégé, it proved possible to convert the FMA from Protégé into OWL DL, while capturing most of its original features. The conversion relies on a set of translation and enrichment rules implemented with flexible options. Unsurprisingly, reasoning with the FMA in OWL proved to be a real challenge, due to its sheer size and complexity, and raised significant inference problems in terms of time and memory requirements. However, various smaller versions have been successfully handled by Racer. Some inconsistencies were identified and several classes reclassified. The results obtained so far show the advantage of OWL DL over frames and, more generally, the usefulness of DLs reasoners for building and maintaining the large-scale biomedical ontologies of the future Semantic Web.
\end{abstract}

\section{Keywords}

Keywords: Anatomy; Foundational Model of Anatomy (FMA); Ontology; Protégé; Web Ontology Language (OWL); Semantic Web

\section{Introduction}

Life sciences have a long tradition of controlled vocabularies. Extensive terminologies, classifications and ontologies have been developed for many years in various biomedical domains. These resources have the potential to contribute to the Semantic Web for Life Sciences, but need to be adapted for it. A large library of biomedical ontologies has been developed in frames, often with Protégé [12]. As OWL is the W3C recommended standard for ontologies [1], converting frame-based ontologies to OWL becomes an important need. Representing ontologies in OWL provides several advantages. Once converted to OWL, ontologies currently developed with frames become easier to integrate with other ontologies and can be used as resources for the Semantic Web. Interoperability of Web ontologies is important for shared use across different biological and medical domains, as expected for example from the Open Biomedical Ontologies (OBO) library. Also of interest is OWL higher 
expressiveness, and precise formal semantics. Another important advantage of OWL is the existence of powerful reasoning services, based on its underlying description logics. Several major ontological and terminological resources in biomedicine have been recently converted to OWL DL, including the Medical Subject Headings (MeSH) [8], the Gene Ontology ${ }^{\mathrm{TM}}$ [9] and the National Cancer Institute Thesaurus [10]. The conversion of other ontologies to OWL has also been investigated, e.g., the UMLS ${ }^{\circledR}$ Metathesaurus ${ }^{\circledR}$ [2] and Semantic Network [11]. Our long term goal is to provide a Web service assisting the conversion of frame-based and OBO ontologies to OWL. Meanwhile, the present study investigates the conversion of a large frame-based ontology into OWL and the reasoning services enabled by this conversion.

The frame-based ontology under investigation is the Digital Anatomist Foundational Model of Anatomy (FMA). It was converted from Protégé 2.1 to OWL DL. The FMA is the most comprehensive ontology of human 'canonical' anatomy [3]. The version used in this study, dated of July 2004, contains 70,169 concepts and more than 1.5 million relations. The FMA was selected for two major reasons. First, anatomy plays a prominent role in biomedicine and many biomedical ontologies and applications refer to anatomical ontologies. As its authors claim, the FMA is " a reference ontology in biomedical informatics for correlating different views of anatomy, aligning existing and emerging ontologies in bioinformatics . .." [3]. Anatomy, together with Gene and Disease reference ontologies, constitute the backbone of the future Semantic Web for Life Sciences. Second, representing the FMA into OWL poses a real challenge from a knowledge representation perspective. It is important to investigate if OWL DL, which is a first order language, has sufficient expressiveness to represent what was originally represented with frames and metaclasses in Protégé. The capacity of OWL editors (e.g., Protégé OWL) and reasoners (e.g., Racer) to deal with the sheer size and complexity of the FMA and the scalability of OWL DL inference techniques to such a large biomedical ontology must be evaluated. Rather than OWL Full used in [5], we selected OWL DL because a main component of our study is to investigate the benefits of a DL representation over frames in terms of reasoning supported by the underlying description logic. OWL DL provides completeness and decidability of the interesting reasoning problems (satisfiability and subsumption) and supports consistency checking and automatic classification. OWL DL reasoners are available (e.g., Racer [16] and Pellet [17]). In contrast, OWL Full is undecidable, offers no computational guarantees and lacks suitable reasoners.

The rest of this paper is organized as follows. The method used to automatically convert the FMA from Protégé 2.1 into OWL DL is first presented (Section 2). Our experience in reasoning with OWL is reported next (Section 3). The choices of conversion, as well as possible perspectives for the FMA and open questions for large-scale ontologies of the future Semantic Web are finally discussed (Section 4).

\section{Conversion to OWL DL}

As DLs and frames share the same object paradigm, it might be thought that converting a Protégé frame-based ${ }^{1}$ ontology into OWL is straightforward and could be achieved by a simple export function mapping Protégé primitives to OWL constructs. But, the export function from Protégé to OWL did not work for the FMA, neither in one step (i.e., directly), nor in two steps (i.e., from database to text then to OWL). Besides, even if it had worked, it would have been ineffective for the following two reasons.

\footnotetext{
${ }^{1}$ In the following, ' Protégé' is used as a shortcut for Protégé-Frames (not for Protégé -OWL). Protégé -Frames (version 2.1) and Protégé -2000 is a tool for building frame-based ontologies. Protégé -OWL (current versions 3.1.1 or 3.2) is a Protégé extension that supports OWL.
} 
First, migrating a frame-based ontology to OWL requires not only a syntactic "translation", but also a semantic "enrichment" [6]. Indeed, property restrictions such as allValuesFrom or someValuesFrom contained in the OWL axioms cannot be directly derived from the original frame representation, where they are not specified. Additionally, classification strongly relies on the logical definition of the classes. A reasoner (e.g., Racer) can only automatically classify classes under "defined" classes ${ }^{2}$, i.e., classes with at least one necessary and sufficient condition. Necessary and sufficient conditions cannot be derived directly from the Protégé model, because, in the frame representation, all slots at class with a specified range or value are considered to be a set of necessary conditions. Specifying defined classes represents a major "enrichment" of the ontology.

The second reason is that the frame-based representation of the FMA in Protégé makes extensive use of metaclasses ${ }^{3}$, which are not allowed in first order languages such as OWL DL. Each anatomical entity is modeled both as a metaclass and as an instance of a metaclass. This was the "technical solution for enabling the selective inheritance of attributes" in Protégé [3] (see Section 4). For example (Table A.1 of Appendix A), Heart is defined as a metaclass, subclass of Organ with cavitated organ parts, itself subclass of Organ, and as an instance of this metaclass. At the meta level, Heart inherits all the slots, facets, characteristics (range, cardinality, inverse, etc.) of its superclasses. Heart inherits from Organ the slot bounded by with multiple values allowed in Surface of organ, the slot arterial supply with multiple values allowed in the classes Artery, Arteriole, Arterial plexus and Set of arteries, the slot venous drainage with multiple values in the classes Subdivision_of_venous_tree_organ and Organ_part_tree_structure, etc. But at the class level, the own slots of Heart are assigned particular values, e.g., bounded by is filled with Surface_of_heart, arterial supply with Right_coronary_artery and Left_coronary_artery, etc. Directly translating metaclasses into OWL would require OWL Full, instead of OWL DL. Simply removing metaclasses as suggested in [5] would not be satisfactory either, since the knowledge encoded at the metaclasses would be lost.

Therefore, we defined our own method of conversion to OWL DL, which aims at both providing the desired enrichments and capturing the knowledge that was encoded at metaclasses, but without resorting such metaclasses.

\subsection{Method of conversion}

The migration was achieved from the CLIPS files generated by Protége when storing the Protégé-frames FMA ${ }^{4}$. The conversion relies on translation (i) and enrichment (ii) rules. Different enrichment rules were defined depending on whether the information was defined at the class or metaclass (iii) level. The conversion rules are implemented with flexible options (the rules used in the current version can be found in the Appendix A).

i. Translation draws on the structural correspondence between Protégé -Frames primitives and OWL constructs. The Protégé class taxonomy defined at meta level is translated into an OWL subclass hierarchy. Template slots defined at the top level are translated into OWL properties with the same features as those specified in Protégé (i.e. same range, inverse, cardinality, etc.), simply mapping each of them to the corresponding OWL primitive (Fig. 1). For example, the Protégé single slots has mass and has boundary, defined with type SYMBOL, allowed values FALSE and

\footnotetext{
${ }^{2}$ Except if a property has a domain (or range) that is a primitive class, which can coerce classes to be reclassified under the primitive class that is the domain or range of the property (Section 4).

${ }^{3} \mathrm{~A}$ metaclass is a class whose instances are themselves classes.

${ }^{4}$ Protégé uses the CLIPS text file format as its default save/load file format for both classes and instances: .pont and .pins files contain respectively domain classes and instances.
} 
$T R U E$, and cardinality 01 , are simply translated into an ow 1 : DatatypeProperty, with range datatype Boolean, and declared to be an owl : FunctionalProperty. The Protégé multislot constitutional part defined with type $S Y M B O L$, allowed parents Physical anatomical entity and inverse slot constitutional part of is translated into an owl:Object-Property with (rdfs:range rdf:resource= "\#Physical anatomical entity") and inverse (owl: inverse of rdf:resource="\# constitutional part of").

ii. Enrichment, in contrast, introduces new logical features. The enrichment rules were designed to reflect the underlying principles of the original FMA model. Some enrichment rules and the rationale behind them are presented below.

2.1.1. Property restrictions-The choice between universal and existential property restrictions is mainly based on the distinct role of template and own slots in Protégé. Template slots " specify which slot each member of a class shall have and what the restrictions (facets) on the values of these slots shall be" [3]. Template slots with their constraints are inherited by the subclasses and the instances. Therefore, allowed parents or allowed classes specified for a template slot at metaclass are converted into universal property restrictions ( owl : allValuesFrom). In contrast, according to the FMA principle of "canonical anatomy" [3], when a class instantiates a metaclass, the specific values assigned to a template slot inherited as own slot describe the typical canonical structure of the particular anatomical entity in terms of relations that should necessarily exist, e.g., in terms of the existing parts composing an organ. Therefore, they are converted into existential property restrictions (owl:someValuesFrom) (Fig. 2).

For example, the multislot bounded by of the metaclass Organ with allowed-parents Surface of organ is converted into the universal restriction ( $\forall$ bounded_by.Surface_of_organ) on the property bounded by of Organ, that is next inherited by its subclass Heart. But when Heart inherits bounded by as an own slot assigned with the value Surface of heart, it is converted into the existential restriction ( $\exists$ bounded_by.Surface_of_heart).

Similarly, venous drainage is restricted by a universal restriction in Heart inherited from its superclasses, but when Heart inherits venous_drainage as an own slot assigned with the values Oblique_vein_of_left_atrium, Left_marginal_vein, Coronary_sinus,

Posterior_vein_of_left_ventricle, Unnamed_tributary_of_cardiac_vein,

Anterior_interventricular_vein, Small_cardiac_vein, etc., they are converted into owl: someValuesFrom restrictions specifying the value constraints on the property for the class Heart (Fig. 3).

2.1.2. Equivalent class definition-A "defined" class has at least one necessary and sufficient condition. At this preliminary step, one slot $p$ is manually selected, and a class $A$ having values $B_{1}, \ldots, B_{n}$ assigned to its own slot $p$ is defined as equivalent to the conjunction of all the existential restrictions on $p$ to the classes $B_{i}$ and of metaclass and superclass of $A$ (after some optimization). As aggregated objects are often described in terms of their parts and as meronymic relationships play a particularly important role in anatomy, we attempted to define anatomical entities in terms of their parts. The property constitutional_part was selected, resulting in 570 defined classes. Thus, the equivalent class expression used for the defined classes combines taxonomic relations, metaclass instantiation and constitutional parts as defined in the original FMA model. For example, the class Heart is defined by the conjunction Organ_with_cavitated_organ_parts $\Pi(\exists$ constitutional_part_Wall_of_heart) П (constitutional_part...), as shown Fig. 4. 
The choice of the property constitutional part was partly motivated by practical issues: constitutional part is relatively well populated in the FMA, compared for instance to custom partonomy, and is therefore computationally more significant. But, such a definition is not "semantically" satisfying for all classes: all anatomical entities cannot be uniformly defined solely in terms of their constitutional parts (the same parts may belong to different structures), and no such constitutional parts are defined for most FMA classes. However, the choice of constitutional part was deemed appropriate for the purpose of investigating reasoning services. Different definitions for the different subtrees, and more complex expressions combining several properties shall be investigated in the future (Section 4).

2.1.3. Metaclasses are converted into ordinary OWL DL classes-First, subclass relations between metaclasses, and meta-class instantiations are both translated into OWL subClassOf axioms ([A] of B in Protégé is converted to A $\sqsubset$ B in OWL). Doing so, the metaclass and instance definitions are merged within a single class in OWL DL (Fig. 5).

Second, the restrictions depend on whether the information was defined at the class or metaclass level, according to the enrichment rules (see (ii) above). Range restrictions of a template slot defined at metaclass are converted to universal property restrictions.

Third, the values of own slots of classes are converted either into existential restrictions on class properties or into values of OWL annotation properties (see Appendix A for details). Structural own slots with values assigned at class are converted to existential restrictions of object or datatype property. Own slots such as definition, name, identifiers (e.g., UWDAID), etc. with values assigned at class level, are converted to owL AnnotationProperty, e.g., definition, prefered name, UWDAID, (Fig. 3) instead of using metaclass instances, which prevents such properties from being propagated to their instances or subclasses.

Thus, each entity of the FMA is represented by a single OWL DL class, with various axioms and annotations. Fig. 5 shows the OWL DL class Heart with its equivalentClass definition combined with subclassof axioms including existential restrictions, derived from the original metaclass and class definitions of Heart in Protégé.

In conclusion, although the original frame-based representation in Protégé makes extensive use of metaclasses, converting the FMA into OWL DL is possible. The OWL version of FMA complies with OWL DL's first order language constraints. In particular a class is not an individual at the same time. In fact, the 'higher' order structure in Protégé frames was removed by replacing metaclass instantiations by subclass axioms. But doing so did not introduce any significant changes, because "all concepts in the FMA Anatomy Taxonomy are subclass of a superclass and also an instance of a metaclass". On the other hand, as explained Section 4.1, it was not necessary to give up some knowledge of the original FMA and nothing has been lost, because the higher expressiveness of OWL DL enabled to capture the intended meanings of the metaclasses.

To compensate for the arbitrariness of some of our choices, the enrichment rules were designed and implemented with flexible options. This permitted to automatically generate various OWL files with different flavors, size and computational complexity. Moreover, these options can be easily modified, which is key to the incremental approach adopted for reasoning (Section 3.1).

\subsection{Results}

In order to minimize the size of the resulting ontology, some inessential laterality distinctions were ignored, i.e. classes differing from their parents only by laterality. The resulting subset comprises about 40,000 concepts, i.e. 57\% of the 70,000 concepts of the original FMA. Applied 
to this subset, the conversion process described earlier resulted in about 117,000 frames, including 40,000 OWL named classes. More precisely, there are 187 properties and 85 individuals specified in this file. 20 properties correspond to annotation, 19 to datatype and 148 to object properties. There are 107,238 subClassOf axioms (38,772 from taxonomy and 3378 from metaclass instantiation), within 39,337 classes where 559 are defined by equivalentClass axioms. OWL constructors allValuesFrom, someValuesFrom, hasvalue, oneof, unionof, FunctionalProperty, SymmetricProperty, Inverseof all occur in the OWL file resulting from the conversion (available at http://mor.nlm.nih.gov/pubs/supp/2005-owled-cg/FMA-constitutionalPartForNS.owl). It took about 15 min to load the FMA OWL file in Protégé -OWL (version $2.1^{5}$ ) on a Windows XP PC with 4 GB of memory (90 min with $512 \mathrm{MB}$ ).

\section{Reasoning with OWL}

Reasoning with OWL proved to be a real challenge, due to the sheer size and complexity of the FMA. Processing the full-fledged FMA in OWL DL raised significant inference problems in terms of time and memory requirements. For this reason, an incremental approach to investigating reasoning services was adopted.

\subsection{Incremental approach}

We used Racer (Version 1.7) with the OWL files generated by the conversion process to investigate consistency checking and automatic classification. Launched from Protégé -OWL, the classification failed for the entire FMA. Running Racer directly from Rice, we experienced problems related to memory limitation (4 GB). Since Racer could not handle the entire FMA OWL file or the smaller file resulting from ignoring laterality distinctions (on our computer), as suggested by the Racer authors, we decided to test smaller versions so as to reduce the size and time issues and to investigate eventual errors, adding more features incrementally. First, a FMA OWL version with all classes but without any properties was checked to test if the taxonomy alone could be successfully classified. Then, we added equivalent class definitions using only one property to test if the ontology with defined classes could pass Racer. Next, we have introduced, step by step, object properties, annotation properties, datatype properties, and finally object properties used for attributed slots. When properties are introduced in partial versions, the conversion rules described previously are applied. For example, a small version where the object property bounds and its inverse bounded by are introduced includes, for each class having these properties specified, the subclass axioms containing the corresponding existential and universal restrictions of the properties bounds and bounded by.

\subsection{Results}

Racer passed the first test: the classification of the FMA OWL version without any properties was successful, taking about 25 min with 512 MB memory on a Pentium 4. Then, the classification with 'defined' classes described by the conjunction of the existential restrictions on the constitutional_part or custom_partonomy property as necessary and sufficient condition was also successful. Next, various versions were generated with all classes but containing a limited number of properties. Depending on the properties introduced and the constructors involved, the tests were successful or not. Some results are summarized below:

Reasoning with Racer was successful for the following partial versions:

1. Ontology with only the class hierarchy defined but without any property.

2. Ontology with defined classes (based on constitutional_part).

\footnotetext{
${ }^{5}$ Four minutes in version 3.1.
} 
3. Ontology with defined classes and annotation properties.

4. Ontology with defined classes, annotation properties, and all datatype properties.

5. Ontology with defined classes and primitive classes with restrictions on the property branch_of in subclassof axioms.

6. Ontology with defined classes and primitive classes with restrictions on the property arterial_supply in sub-Classof axioms.

7. Ontology with defined classes and primitive classes with restrictions on the property 2D_part in subclass axioms.

8. Ontology with defined classes and primitive classes with restrictions on the property bounds and its inverse bounded_by in subclass axioms.

9. Ontology with defined classes and primitive classes with restrictions on properties dimension and has_physical_state in subclass axioms.

10. Ontology with primitive classes with restrictions on attributed slot location and all slots used in location (e.g., related_object, etc.).

11. Ontology with primitive classes with restrictions on attributed slot attributed_part and all slots used in attributed_part (e.g., related_part, etc.)

Reasoning with Racer failed for:

12. Ontology with defined classes and annotation properties, with the addition of primitive classes with restrictions on all the object properties in subclass axioms.

13. Ontology with defined classes and primitive classes with restrictions on all object properties in subclass axioms.

14. Ontology with primitive classes with restrictions on the property branch_of and its inverse in subclass axioms.

15. Ontology with subclass axioms with restrictions on the property continuous_with, declared symmetric.

The results of the tests are summarized in two tables, showing the OWL DL constructs (Table 1) and axioms (Table 2 ) involved in each test (presence denoted by ' $\bullet$ '). $A$ is a class name, $C$ is a class, $o$ is an individual name, $R$ is an object property, $T$ is a datatype property, $D$ is a data range, $v$ is a data value, and $l, m$ and $n$ are integers (following usual notations borrowed from OWL authors [1]). Annotations ( AnnotationProperty) are omitted in the tables, but tests \#3, \#4 and \#12 include them. As there was no hierarchy of relationships specified in the original frame-based FMA, equivalent or sub-property axioms including equivalentProperty and subPropertyof were not defined in our conversion.

The reasons for failure are not easy to analyze. For instance, Racer was successful for some tests including inverse properties, such as test \#8 having equivalent class axioms based on constitutional_part and restrictions on the property bounds and its inverse bounded_by, and for a test with constitutional_part and its inverse constitutional_part_of. However, it failed for some tests including inverses, such as test \#14 having restrictions on the property branch of and its inverse branch. This test, however, does not use any nominal, cardinality or functional axioms. This experiment shows that the sheer size of the FMA is not the only issue. The results of reasoning with OWL for the FMA are related to several factors, including the complexity of the ontology generated due to the OWL constructors used, i.e. the presence of nominals (e.g., oneOf), of inverseof axioms or 
"global" axioms, etc. and their interactions. The origin of the computational difficulties encountered is not completely clear at the moment. On the one hand, some inference problems might come from imperfect conversion. On the other hand, theoretical work has proved that reasoning is NExpTime-complete for OWL DL $(\operatorname{SHOIN}(D))$, and precisely inverse, nominals, number restrictions, anonymous classes all occur in the FMA ontology in OWL. The algorithms and optimization techniques implemented by the reasoners, are certainly critical issues for the FMA.

\subsection{Benefits}

Although problems with computational resources occurred while reasoning with the fullfledged FMA in OWL DL, Racer could handle various less complex versions, which still enabled the detection of inconsistencies in the original FMA and the reclassification of some classes.

No inconsistencies were found in the first versions tested, but when datatype properties were added, several inconsistencies were identified. One hundred and thirteen classes were identified as unsatisfiable by Racer because of opposite boolean values:

- Inconsistencies from conflicts between metaclass and class definitions in Protégé. A class having a boolean value in its own slot and which inherits the opposite value from its superclasses is unsatisfiable in OWL. For example, zone_of_cell is unsatisfiable (hence, so are all its subclasses) because the own slot has_mass was assigned FALSE at instance (converted to the restriction has_mass: false), while this single-slot had value TRUE at its superclass Material physical anatomical entity (converted to has_mass:true). Other inconsistencies were revealed from the inconsistency of the metaclass and class definitions of an entity. A class $A$ subclass of $B$ and instance of $C$ in FMA, where $B$ and $C$ have opposite values for a boolean datatype property (e.g., has_mass) is unsatisfiable in OWL. For example, in Protégé Compartment subdivision is defined as a subclass of Anatomical cluster, which is a subclass of Material_physical_anatomical_entity (has mass:TRUE). On the other hand, Compartment_subdivision is an instance of Anatomical space, which is a subclass of Non- material_physical_anatomical_entity (has mass: FALSE).

- Inconsistencies from conflicting global and local domain (or range). rdfs: range (resp. domain) restrictions are global. Thus if $p$ has class $A^{\prime}$ as domain and $B^{\prime}$ as range, and $A$ has a property $p$ with range $B$, then $B$ must be a subclass of $B^{\prime}$ and $A$ must be a subclass of $A^{\prime}$. Conflicting definitions of global and local ranges or domains lead to inconsistencies in OWL. For example, Surface_of_wrist is unsatisfiable because its 2D_part has an existential restriction to Anatomic_snuff_box which is a subclass of Material_physical_anatomical_entity (has mass:TRUE), while the range of 2D_part is Non-material_physical_anatomical_entity (has mass:FALSE). These inconsistencies exhibit modeling errors in the original Protégé FMA.

Racer also reclassified some classes. In the ontology including defined classes based on the constitutional part property, 286 classes of the asserted hierarchy were moved within the inferred hierarchy and some classes were identified to be equivalent. For example, as the two sibling classes Wall of biatrial part of heart and Wall of biventricular part of heart have the same constitutional parts 6 in the original FMA, they became equivalent for this definition.

\footnotetext{
${ }^{6}$ Fibroelastic connective tissue of endocardium, Fibrocollagenous sheath of cardiac muscle tissue and Fibroelastic connective tissue of epicardium.
} 
However, the equivalence did not hold anymore when adding other restrictions to these definitions. For example, adding restrictions on the property constitutional_part_of enables to differentiate the two classes, as they are parts of different wholes: Wall of biventricular part of heart is a constitutional part of Biventricular part of heart, while Wall of biatrial part of heart is a constitutional part of Biatrial part of heart. Thus, although most occurrences of reclassification were related to the class definitions in terms of their constitutional parts, this example nevertheless shows the power of reasoning with OWL DL.

In conclusion, the results obtained so far illustrate the benefits of representing the FMA in OWL DL. First, checking the logical consistency of the FMA enabled the identification of errors that would probably have been missed otherwise. Second, automatically computing the classification hierarchy is another advantage for such a large ontology. As the FMA has been under development at the University of Washington since 1994 and is still evolving, such services are very useful for quality assurance purposes.

\section{Discussion and perspectives}

This study is a first step towards the representation of the FMA in OWL. Several issues remain open and different perspectives shall be considered in the future.

\subsection{Using OWL DL or OWL Full?}

A recent study [5] proposes to translate the FMA into OWL Full, pointing out both computational and representational issues: "The conversion from frames to $O W L D L$ required us to forgo representing some features of the FMA such as metaclasses ... OWL DL representation is possible, but requires to give up some of the original features". The authors advocate selecting OWL Full for the FMA and OWL DL only for applications, suggesting a two-layered approach: "The first layer consists of a generic conversion tool that generates a representation of the FMA in OWL Full. The second layer consists of several application specific optimization tools that simplify the OWL Full representation of concepts into OWL $D L$ ones by removing all the features unnecessary according to the application context".

In contrast, we propose to use OWL DL for representing the FMA, and, more generally, reference ontologies. This work shows that converting the FMA from its frame-based representation in Protégé into OWL DL is indeed possible and succeeds in capturing most features of the original FMA. We converted the FMA into OWL DL with all the knowledge encoded at its metaclasses, and our conversion still complies with OWL DL constraints. In particular, no entity is at the same time both a class and an individual. The original direct subclasses, superclasses, template slots, slot-constraints, defined at Protégé metaclasses are translated using OWL DL constructs and axioms. The main transformation that enabled the use of OWL DL is the deletion of the Protégé higher order structure, achieved by replacing metaclass instantiations by subclass axioms. But this transformation did not introduce significant changes, since the class and the metaclass hierarchies were integrated in the original model: "except for its root, all concepts in the Anatomy Taxonomy are subclass of a superclass and also an instance of a metaclass" [3]. As shown below, OWL DL's higher expressiveness enables to capture most intended meanings of the Protégé metaclasses (with the exception of "set of sets", which does not represent a significant loss in our opinion, considering their use in Protégé). In fact, metaclasses were used for the following reasons [3,4]:

1. Metaclasses are used to model a given anatomical entitiy as a "set of sets", (e.g., Vertebra as a set of different types of vertebrae: cervical, thoracic, lumbar, themselves sets of other sets, e.g., first, second, ..., fifth lumbar vertebra). A first order language such as OWL DL cannot capture this feature. However, the use of the representation of an entity as a "set of sets" is quite limited in Protégé. In fact, the " members of each 
of these collections are represented in Protégé as subclasses of Vertebra" [3], e.g., "the class Vertebra subsumes different collection of vertebrae, cervical, thoracic, and lumbar vertebra", which are further refined into more specialized subclasses.

2. Metaclasses are also used "to enforce slot value restrictions" [4]. In frames, a slot inherited can only be refined to subclasses of its initial range. For example, when Cervical Vertebra inherits from Vertebra the slot part of with range Vertebral Column, its range must be a subclass of Vertebral Column. Metaclasses were intended to enforce restrictions to other classes, such as class Cervical Vertebral Column, which is not a subclass of Vertebral Column in the FMA model, but a part of it. Thanks to metaclass instantiation, the appropriate values are assigned to own slots at class (Section 2.1). This feature is no longer needed in OWL, since it is possible to use subclass of axioms with various restrictions instead, e.g., $\exists$ part_of

Vertebral_Column for the class Vertebra and $\exists$ part_of

Cervical_Vertebral_Column for its subclass Cervical_Vertebra, although

Cervical_Vertebral_Column is not subsumed by Vertebral_Column.

3. Metaclasses were also intended to specify multiple values specific to each class, e.g., specifying that a Vertebra has parts Body of vertebra, Vertebral arch, Bone of vertebra, etc. In OWL, this can be captured by the conjunction of several restrictions such as $\exists$ part.Body_of_vertebra) $\Pi(\exists$ part.Vertebral_arch) $\Pi$ ( $\forall$ part. Bone_of_vertebra), etc.

4. Finally, metaclasses are used for specifying metadata such as name, author, authority, UWDAID, etc. Assigning values to these 'non structural' own slots at metaclass instantiation prevents them from being propagated to their instances or subclasses. This can be obtained in OWL in using AnnotationProperty.

The main reason for selecting OWL DL over OWL Full is that OWL DL is decidable and supports powerful reasoning services such as consistency checking and automatic classification. OWL DL reasoners are available (e.g., Racer [16], Pellet [17]). In contrast, OWL Full is undecidable, offers no computational guarantees and lacks suitable reasoners. A reference ontology is generally a large ontology intended to provide a controlled vocabulary for a domain. Thus, it is crucial to guarantee the quality and correctness of such reference ontologies. Automated reasoning services are precious for quality assurance of ontologies. Current results are encouraging and the computational difficulties encountered during this study will undoubtedly be overcome soon. In any case, as shown in Sections 3.2-3.3, the results inferred from reasoning even on partial versions are helpful for improving the consistency of the entire ontology.

\subsection{Scalability of reasoning}

The size and complexity of the FMA raise important computational issues. As far as we know, the NCI Thesaurus was one of the largest file in Protégé OWL so far. But it is much smaller and exhibits less complexity than the FMA in OWL. The NCI Thesaurus contains 53,000 frames, including 34,000 classes, 100 properties and 9000 conditions, while the original FMA contains 70,000 concepts and the converted subset 117,000 frames, including 40,000 OWL classes, 187 properties and about 110,000 axioms. But the most important difference is the language complexity. The NCI Thesaurus was converted to OWL Lite, while the FMA is represented in OWL DL. The OWL DL constructs (Table 1) and axioms (Table 2) show that the complexity of the FMA is much higher than NCI. The size and complexity of the FMA in OWL make it a real challenge for DLs systems. The current tests done with the FMA show that, with the current state of the art of DL inference technology, such complexity might cause significant inference problems in terms of time and memory requirements. Indeed, the main problem was computational. Some optimizations were devised to reduce the complexity of the 
file generated. For example, it was necessary to reduce the number of disjunctions generated by the conversion for the domain of properties, which otherwise caused Racer - or any inference system - to run into memory problems. Interestingly, after optimization, two classes remain in the domain of location instead of 1618 originally [15]. Difficulties also occurred with inverse properties. However, Racer successfully handled various less complex versions of the FMA in OWL DL, detected inconsistencies, and reclassified classes. This experiment was done with Racer version 1.7. As Racer evolves - for example, its authors are currently working on optimizations that address the issue of inverse roles - it will be worthwhile to repeat these tests. Analogously, we would like to evaluate the performance of other OWL DL reasoners (e.g., Pellet [17] Fact++ [18]). The results obtained with RacerPro ${ }^{\mathrm{TM}}$ [16] are encouraging so far. The new decision procedures and optimizations being currently implemented in highly optimised DL reasoners, e.g., Fact++ [18] may also improve the results in the near future.

\subsection{Domain and application ontologies}

A reference ontology such as the FMA, is a domain ontology supposed to be applicationindependent. Applications most often do not require the whole FMA but only specific modules extracted from it. What is more problematic is that different applications may require different class definitions, because of their specific goals. Indeed, results of reasoning with OWL DL strongly rely on the defined classes expressions, and the intended use of the ontology biases the necessary and sufficient conditions from what is to be achieved via classification or instance recognition. For example, the Brain Cortex Anatomy application [7] only needs concepts involved in Brain anatomy and instance recognition of some entities is mainly based on their definitions in terms of boundaries. Other applications such as the Virtual Soldier project may require concepts involved in the neighborhood of the heart and perhaps mainly definitions based on parts. Hence, the question that arises is whether it is achievable to have 'applicationindependent' class definitions for a reference ontology such as the FMA.

In fact, several possible options might be considered for the defined classes of the FMA. (1) Each class has a single definition, based on the conjunction of all the qualified property restrictions derived from the values of its own structural slots and attributed relations; (2) each class has a set of several equivalent definitions; (3) each class has one preferred definition, the other conditions being simply necessary; (4) there are no a priori "defined" classes, but only primitive OWL DL classes, all axioms expressing only necessary conditions. As the FMA aims at being a "shared reference ontology", its representation in OWL DL might be considered as a first formal specification, to be further refined into more detailed formal specifications for each application, by adding relevant axioms. Therefore, the best options to consider for largescale domain ontologies such as the FMA might be to represent them in OWL DL either with a preferred definition (3) or with only primitive classes (4), but with a library of optional equivalent class definitions, the validity of which having been checked. Then, more specific OWL DL ontologies may be further customized for particular applications, e.g., by adding relevant equivalent class axioms (selected from the library or created specifically). The advantage of this solution is twofold. First, it would concretely implements the notion of a "Semantic Web reference ontology" specified independently of applications. Second, it allows users to benefit from DL reasoning services such as consistency checking and classification for both the general reference ontology and the more customized ones.

\subsection{Future work}

The next step is to improve the current conversion so as to provide a more reliable and enriched representation of the FMA in OWL. A possible direction is to introduce additional enrichment to the FMA original model mainly by adding qualified number restrictions, disjointness and 
closure axioms (Section 4.4.1) and by creating reliable formal definitions for the classes (Section 4.4.2).

4.4.1. Adding qualified number restrictions, disjointness and closure axiomsIn the future, we would like to improve the current conversion process and to remove some of its limitations:

- First, we suggest adding disjointness axioms between the relevant classes. Ideally, a classification satisfies the so-called "jointly exhaustive and pairwise disjoint" rule. The inconsistencies reported Section 3 are mainly based on opposite values of a boolean datatype property and their propagation, but disjointness axioms will most probably lead to identifying more inconsistencies in the FMA.

- Second, we propose to use qualified cardinality restrictions. We converted structural own slots values into existential property restrictions mainly for two reasons. On the one hand, the assumption that if a class $A$ has a slot $p$ filled with values $B_{1}, B_{2}, \ldots$ $B_{n}$ in Protégé (e.g., constitutional part), an implicit assumption is that for every individual of $A, p$ has at least one value of each class $B_{i}$. On the other hand we were confronted to the limited expressiveness of OWL, which does not support qualified cardinality restrictions. However, defining restrictions such as "hasPart someValuesFrom $B_{1}$ " and "hasPart someValueFrom $B_{2}$ " is weaker than "hasPart exactly one $B_{1}$ and one $B_{2}$ ", as it does not prevent from having several parts of the same $B_{i}$. If the next version of OWL supports qualified cardinality restrictions, more precise definitions might be provided.

- Third, we suggest completing our current class definitions by so-called closure axioms [13]. In fact, neither existential property restrictions nor qualified cardinality restrictions prevent properties from being assigned values from an unwanted class. In contrast, adding an allValuesFrom restriction to the class $B_{1} \sqcup B_{2}$ would coerce values to come only from $B_{1}$ or $B_{2}$.

Qualified cardinality and closure axioms would allow to represent more accurately the original FMA definitions, either given in natural language or implicit in Protégé frames. For example, the equivalent class definition Left_lung $=$ Lung $\equiv \Pi(=1$

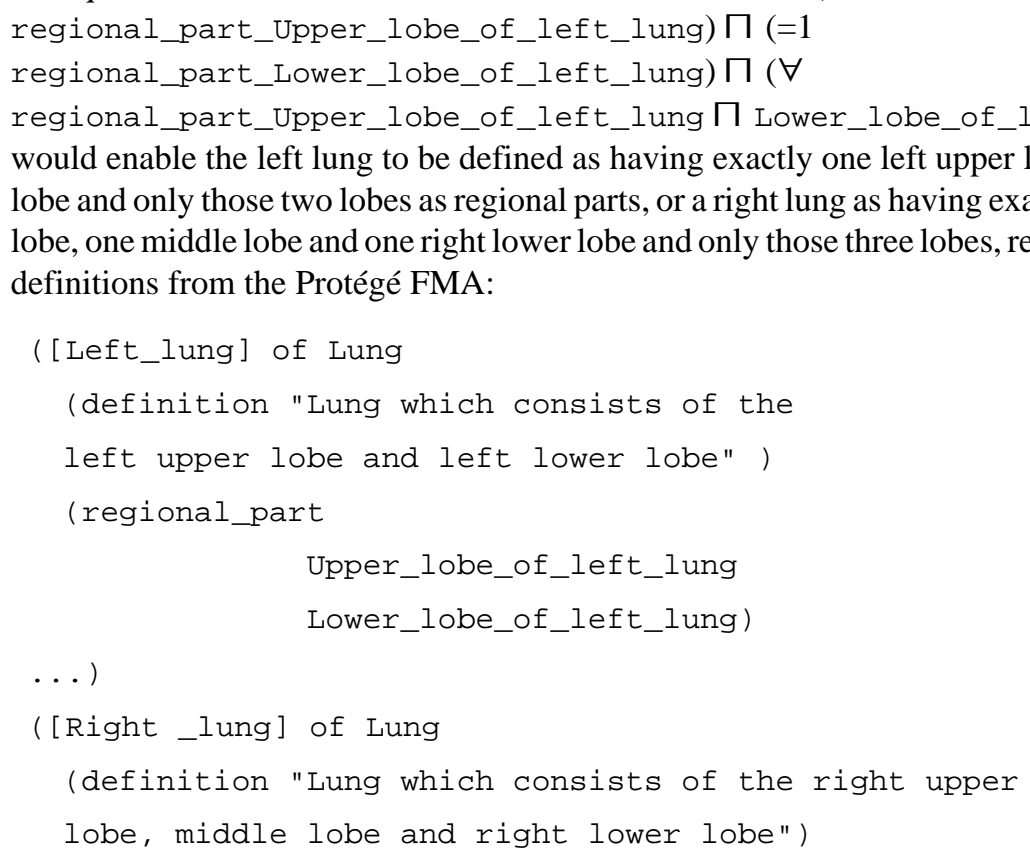
definitions from the Protégé FMA: would enable the left lung to be defined as having exactly one left upper lobe, one left lower lobe and only those two lobes as regional parts, or a right lung as having exactly one right upper lobe, one middle lobe and one right lower lobe and only those three lobes, reflecting the original 


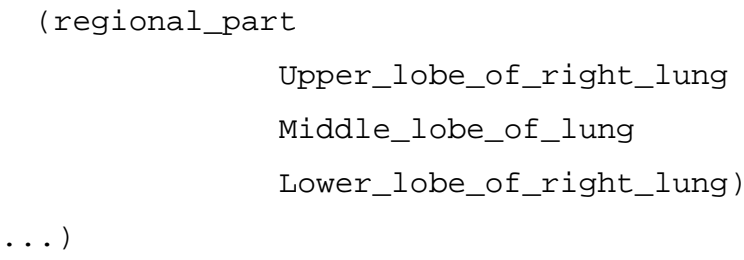

4.4.2. Enriching the FMA with formal definitions-Currently, the biggest challenge in converting the FMA from its original Protégé frame-based representation to OWL DL is certainly to specify explicit and precise formal definitions for the classes, i.e., necessary and sufficient conditions. In the current experimental version, the equivalent class expressions used to define classes combine taxonomic relations, metaclass instantiation and constitutional parts that were defined in the original FMA model. Only one property, constitutional_part (or custom_partonomy) was selected for the equivalent class definitions. This simple method is not satisfying in many respects and is a serious limitation for the results obtained from reasoning, especially from classification. Defining anatomical entities solely on the basis of their constitutional parts is not semantically correct for all classes, and no such constitutional parts are defined for most FMA classes. Defining classes from their constitutional parts may perhaps be appropriate for Organ, but other anatomical entities might require different criteria of identification (e.g., Organ part, Cell, or Tissue). As all anatomical entities do not share the same kind of definition, different expression templates should be specified for the different subtrees (e.g., Organ, Cell, etc). Equivalent conditions - single or multiple, default or optional - must be defined in close collaboration with the FMA authors, so as to have "semantically" correct expressions supporting the unique identification of anatomical entities. Conversion rules should be modified to accommodate arbitrary combinations of properties, constructors, and cardinality restrictions and to support specific expressions for each subtree.

In this first attempt, the conversion aimed at capturing the features of the Protégé representation of the FMA as faithfully as possible, in order to evaluate its original properties. In the future, in addition to above proposals, we suggest to introduce some changes in the model. For example, the OWL classes used for the Protégé attributed relations might be specified by $n$ ary relations in an external database related to the ontology. New classes might be defined for organizational and consistency reasons, e.g., Venous_drainage, Arterial_Supply, etc.

\subsection{Conclusion}

Converting the whole FMA from its original frame-based representation into the first order language OWL DL was possible, and most features of the original FMA model were captured. With the current state-of-art of DL reasoners, reasoning with OWL proved to be a real challenge, because of the sheer size and complexity of the FMA in OWL. Reasoning with the full-fledged FMA raised computational difficulties, but after some optimizations, various smaller versions were successfully tested with Racer. Several inconsistencies were revealed in the original modeling of the FMA. Some classes of the asserted hierarchy were reclassified and some classes identified to be equivalent. Although most of them were related to the definition of the anatomical entities in terms of their constitutional parts, this example illustrates the power of reasoning with OWL DL. The results obtained so far demonstrate the advantages of OWL over frames for large domain ontologies of the future Semantic Web such as the FMA. However, this experiment is only a first step and the current versions are not satisfactory in many respects. In particular, the experimental method used for creating defined classes from their constitutional parts has to be revised. Correct expressions supporting the unique identification of anatomical entities shall be defined. The conversion rules shall be modified and refined. Tests with RacerPro ${ }^{\mathrm{TM}}$ are encouraging. Other reasoners also need to be tested. 
OWL semantics and DL techniques have the potential of providing a significant help in enriching and improving the FMA ontology for the future Semantic Web for Life Sciences.

\section{Acknowledgments}

This research was supported in part by the Intramural Research Program of the National Institutes of Health (NIH), National Library of Medicine (NLM), while Pr. Christine Golbreich and Dr. Songmao Zhang were visiting scholars at the Lister Hill National Center for Biomedical Communications, NLM, NIH. Our thanks to Volker Haarslev and Ralf Moller for their valuable advice and help on OWL and Racer, to Cornelius Rosse, Jose Mejino and Todd Detwiler for the FMA, and to other helpful advice.

\section{References}

1. Dean, M.; Schreiber, G.; Bechhofer, S.; van Harmelen, F.; Hendler, J.; Horrocks, I.; McGuinness, DL.; Patel-Schneider, PF.; Stein, LA. OWL web ontology language reference, W3C Recommendation. 2004. available at http://www.w3.org/TR/owl-ref/

2. Cornet R, Abu-Hanna A. Usability of expressive description logics—a case study in UMLS. Proc AMIA Symp 2002:180-184. [PubMed: 12463811]

3. Rosse C, Mejino JL Jr. A reference ontology for biomedical informatics: the Foundational Model of Anatomy. J Biomed Inform 2003;36(6):478-500. [PubMed: 14759820]

4. Noy N, Musen MA, Mejino JL, Rosse C. Pushing the envelope: challenges in a frame-based representation of human anatomy. Data Knowledge Eng J 2002;48(3):335-359.

5. Dameron O, Rubin DL, Musen AM. Challenges in converting frame-based ontology into OWL: the Foundational Model of Anatomy Case-Study. AMIA Annu Symp Proc 2005:181-185. [PubMed: 16779026]

6. Golbreich, C.; Zhang, S.; Bodenreider, O. Migrating the FMA from Protégé to OWL; Eighth Protégé International Conference; Madrid. 2005. p. 108-111.

7. Golbreich, C.; Dameron, O.; Bierlaire, O.; Gibaud, B. Proceedings of the workshop “OWL Experiences and Directions". Galway, Ireland: [November 11-12, 2005]. What reasoning support for Ontology and Rules? The brain anatomy case study. electronic proceedings: http://ceur-ws.org/

8. Soualmia, L.; Golbreich, C.; Darmoni, S. Representing the MeSH in OWL: Towards a semiautomatic migration; Proceedings of the KR 2004 Workshop on Formal Biomedical Knowledge Representation. 2004. p. 81-87.Available at http://sunsite.informatik.rwthaachen.de/Publications/CEUR-WS//Vol-102/soualmia.pdf

9. Wroe CJ, Stevens R, Goble CA, Ashburner M. A methodology to migrate the Gene Ontology to a description logic environment using DAML + OIL. Pac Symp Biocomput 2003:624-635. [PubMed: 12603063]

10. Golbeck J, Fragoso G, Hartel F, Hendler J, Oberthaler J, Parsia B. The National Cancer Institute's thesaurus and ontology. J Web Semantics 2003;1(1):75-80.

11. Kashyap, V.; Borgida, A. Representing the UMLS Semantic Network using OWL: (Or "What's in a Semantic Web link?”). In: Fensel, D.; Sycara, K.; Mylopoulos, J., editors. The SemanticWebISWC. Springer-Verlag; Heidelberg: 2003. p. 1-16.

12. Noy NF, Sintek M, Decker S, Crubezy M, Fergerson RW, Musen MA. Creating semantic web contents with protege-2000. IEEE Intell Syst 2001;16(2):60-71.

13. Rector, A.; Drummond, N.; Horridge, M.; Rogers, J.; Knublauch, H.; Stevens, R.; Wang, H.; Wroe, C. OWL Pizzas: practical experience in teaching OWL-DL: common errors and common patterns; European Conference on Knowledge Acquisition; 2004. p. 63-81.

15. Zhang, S.; Bodenreider, O.; Golbreich, C. Pac Symp Biocomput. 2006. Experience in reasoning with the Foundational Model of Anatomy in OWL DL; p. 200-211.PMID: 17094240

16. RacerPro. http://www.franz.com/products/racer/

17. Pellet OWL reasoner. Maryland Information and Network Dynamics Lab. 2003. http://www.mindswap.org/2003/pellet/index.shtml

18. Tsarkov D, Horrocks I. Efficient reasoning with range and domain constraints. Proceedings of DL 2004:41-50. 


\section{Appendix A. Conversion rules}

The rules depend on whether the information is stored at metaclass or class level. They capture all the knowledge of the original Protégé model, defined either at metaclasses, classes, or instances, while respecting the original principles of the FMA.

\section{A.1. Class information}

Classes and slots - stored at (meta)class level in Protégé - are converted into OWL classes and properties with specified domain ( $r d f s:$ domain) and range ( rdfs:range). The property characteristics are translated to OWL corresponding constructs: inverse to owl: inverseof, logical characteristics, i.e. symmetric to owl : Symmet ricProperty, property cardinality and values restrictions to owl:FunctionalProperty and owl : hasValue. In practice, the rules are the following:

\section{Top-level slots}

specified in Protégé to save top-level slot information, are converted to DatatypeProperty or ObjectProperty with the corresponding range and restrictions, according to their definition. For example,

1. top-level slot with type $S Y M B O L$ and allowed-values TRUE and FALSE is converted to a DatatypeProperty with range \#Boolean, e.g., has boundary (Table A.1 Example \#1);

2. top-level slot with type $S Y M B O L$ with allowed-values different from TRUE or FALSE is converted to an ObjectProperty with an enumerated class oneof \{allowed-values\} as range;

3. top-level slot with type $S Y M B O L$ with allowed-classes (allowed-parents) is converted to an ObjectProperty with the union of the allowed (meta)classes as range, e.g., the range of the multislot venous drainage is the unionof

\#Subdivision_of_venous_tree_organ and \# Organ_part_tree_structure (Example \#2);

4. top-level slot with type INSTANCE is converted to an ObjectProperty

Single-slot-with cardinality 01 is converted to FunctionalProperty (Example \#1).

Inverse-slot-If a top level slot has an 'inverse-slot', it is converted to SymmetricProperty or Inverseof: if the inverse value is itself, it is SymmetricProperty with range assigned to its domain, otherwise it is Inverseof. Thus, for example, the top level slot has boundary is converted to a Dat at ypeP roperty with range \#boolean, with a FunctionalProperty restriction, while bounded by is converted to an ObjectProperty with range \#Physical_anatomical_entity, and inverseof \#bounds (Example \#3).

\section{Slots at class}

enable to define the domain of an OWL property and to refine its value constraints: if $\mathrm{p}$ is slot of class $\mathrm{C}_{1}$, then $\mathrm{C}_{1}$ becomes the domain of $p$, e.g., \# Physical_anatomical_entity becomes the domain of has_boundary (Example \#1); if the same slot p occurs in class $\mathrm{C}_{2}$, then the domain of $\mathrm{p}$ is the union of $\mathrm{C}_{1}$ and $\mathrm{C}_{2}$ (e.g., the domain of bounded_by in Example \#3); Optimization of domain $\mathrm{C}_{1} \sqcup \mathrm{C}_{2} \ldots \sqcup 3 \mathrm{C}_{n}$ has been done: if $\mathrm{C}_{i}$ is descendant of another 
class according to two levels of is-a, then $\mathrm{C}_{i}$ is removed from the domain (reducing so "arterial supply" domain from 4007 classes to 4 !).

Allowed-parents, allowed-classes-value define the allowed values of properties at class. They are converted to necessary conditions expressing value constraints on the property for this class: if $\mathrm{p}$ is slot of class $\mathrm{C}$ specified with allowed-parents or allowed-classes (respectively with value), then $\mathrm{p}$ is converted by a necessary condition at class $\mathrm{C}$ expressing value constraints for $p$ by owl : allValuesFrom the union class of all its allowed-parents or allowed-classes, e.g., allowed-parents Surface of organ or allowed-parents Subdivision of venous tree organ Organ part tree structure (Example \#4) (respectively by hasValue).

Is-a-is converted into subsumption axioms ( subclassof): A is-a B (if B is not USER nor:STANDARD-CLASS or:STANDARD-SLOT or RELATION) is converted to A subClassOf B (resp. A is-a B1 and A is-a B2 is converted to subclassof B1 $\Pi$ subclassof B2), e.g., Organ is-a Anatomical structure (Example \#4).

\section{A.2. Instance information}

The values of own slots of classes - specified at instance level in Protégé to store data specific to a class - are converted either into OWL values of annotation properties or into existential restrictions on the class properties. In practice, the rules are the following:

- Non-structural slots. In Protégé non structural slots such as 'preferred name', 'synonyms', 'definition', 'UWDAID', 'author' etc., are defined as slots of metaclasses. When classes instantiate the metaclasses, they become own slots assigned with values specific to each class, which are thus not propagated to their instances or subclasses [4]. For example, UWDAID value for Heart is 7088. We used OWL annotations on classes instead, which are allowed in OWL DL under some constraints, e.g., the AnnotationProperty UWDAID (Table A.2). Properties designed as AnnotationProperty have been manually selected. They include Preferred name, Synonyms, UWDAID, author, authority, modification, name, Date entered modified, TA ID, definition, modification, 'Latin name TA', UMLS ID, Outdated meaning, ther Latin equivalents, Source, View, Abbreviation English equivalent, etc.

- Structural slots. Another use of metaclass in Protégé is for "structural" slots, such as part of, custom partonomy, bounded by, arterial supply, etc. It enables to specify each class for "canonical" anatomy with particular values assigned to its own slots, which are thus not propagated.

For example, a 'canonical' Heart is specified having a Right atrium, a Left atrium, a Right ventricle, a Left ventricule as custom partonomy, being bounded by a Surface of heart.

Structural own slots are converted to necessary (or necessary and sufficient) conditions at class C asserting someValues-From constraints for $\mathrm{p}$ to the union of all the classes assigned to $\mathrm{p}$. For example bounded_by Surface_of_heart is converted to a someValuesFrom restriction on the property \# bounded_by, expressing that any instance of heart is necessarily bounded by at least one \#Surface_of_heart (Table A.3).

- Attributed relations. The values of attributed relations are represented in OWL by nested classes generated in following the same conversion rules as for classes.

For example attributed part is an attributed relation which allowed values are instances of class Part_of_relationship_value, e.g., fm-live 12491, fm-live 12492, etc. They are converted to someValuesFrom restrictions on the property \#attributed_part (Table A. $4)$. 
- Instantiation of metaclasses. Metaclass instantiation is replaced by subsumption axiom using subclassof axioms ${ }^{7}$ : [A] of B is converted to a subclassof axiom A B , e.g., the axiom of class Heart subclassof_Organ_with_cavitated_organ_parts (Table A.3).

- Generating individuals in OWL DL. Based on the slot dimension presented earlier, one individual ${ }^{8}$ is generated under owl: Thing for each allowed value of this slot, as shown in Table A.5.

Additional examples illustrating the conversion rules are available online at http://mor.nlm.nih.gov/pubs/supp/2006-psbsz/2006-psb-sz-supp.pdf.

${ }^{7}$ For " $[\mathrm{A}]$ of $\mathrm{A}$ ", " $[\mathrm{A}]$ of B " when "A is-a B “, or " $[\mathrm{A}]$ of B " when "A is-a C" and C is a descendant of B, some optimizations prevent the generation of useless axioms.

${ }^{8}$ Individuals are prefixed by "individual “, because some allowed-values of slots share names with classes in the FMA in Protégé, such as Inferior and Liquid. 


\begin{tabular}{|c|c|}
\hline Protégé slot & OWL property \\
\hline Type INTEGER, FLOAT, STRING & $\begin{array}{l}\text { DatatypeProperty with range datatype integer, } \\
\text { float and string }\end{array}$ \\
\hline $\begin{array}{l}\text { Type } S Y M B O L \text { with allowed values TRUE or } \\
\text { FALSE }\end{array}$ & DatatypeProperty with range datatype boolean \\
\hline $\begin{array}{l}\text { Type } S Y M B O L \text { with allowed values (other } \\
\text { than TRUE or FALSE) }\end{array}$ & $\begin{array}{l}\text { ObjectProperty with range the enumerated class of } \\
\text { all the allowed individuals }\end{array}$ \\
\hline Type $S Y M B O L$ with allowed parents & \multirow{2}{*}{$\begin{array}{l}\text { ObjectProperty with range the union of all the } \\
\text { allowed classes }\end{array}$} \\
\hline Type INSTANCE with allowed classes & \\
\hline
\end{tabular}

Fig. 1.

Some translation rules for slots. 


\begin{tabular}{|l|l|l|}
\hline \multicolumn{2}{|c|}{ Protégé slot } & \multicolumn{1}{c|}{ OWL property restriction } \\
\hline \multirow{4}{*}{$\begin{array}{l}\text { Protégé template slot at } \\
\text { metaclass }\end{array}$} & $\begin{array}{l}\text { Slot with allowed parents or } \\
\text { allowed classes } C_{i}\end{array}$ & $\begin{array}{l}\text { Owl: allValues From property } \\
\text { restriction constrained to the union of } \\
\text { all the classes } C_{i}\end{array}$ \\
\cline { 2 - 3 } & Slot with an allowed value & $\begin{array}{l}\text { owl:hasValue property restriction } \\
\text { constrained to the specified value }\end{array}$ \\
\hline \multirow{4}{*}{ Protégé own slot at class } & $\begin{array}{l}\text { Slot assigned with a specific } \\
\text { class } C \text { as value }\end{array}$ & $\begin{array}{l}\text { owl: someValues From property } \\
\text { restriction constrained to the class } C\end{array}$ \\
\cline { 2 - 3 } & $\begin{array}{l}\text { Slot assigned with a specific } \\
\text { datatype or individual value }\end{array}$ & $\begin{array}{l}\text { owl }: \text { hasValue property restriction } \\
\text { constrained to the specified value }\end{array}$ \\
\hline
\end{tabular}

Fig. 2.

Some enrichment rules. 


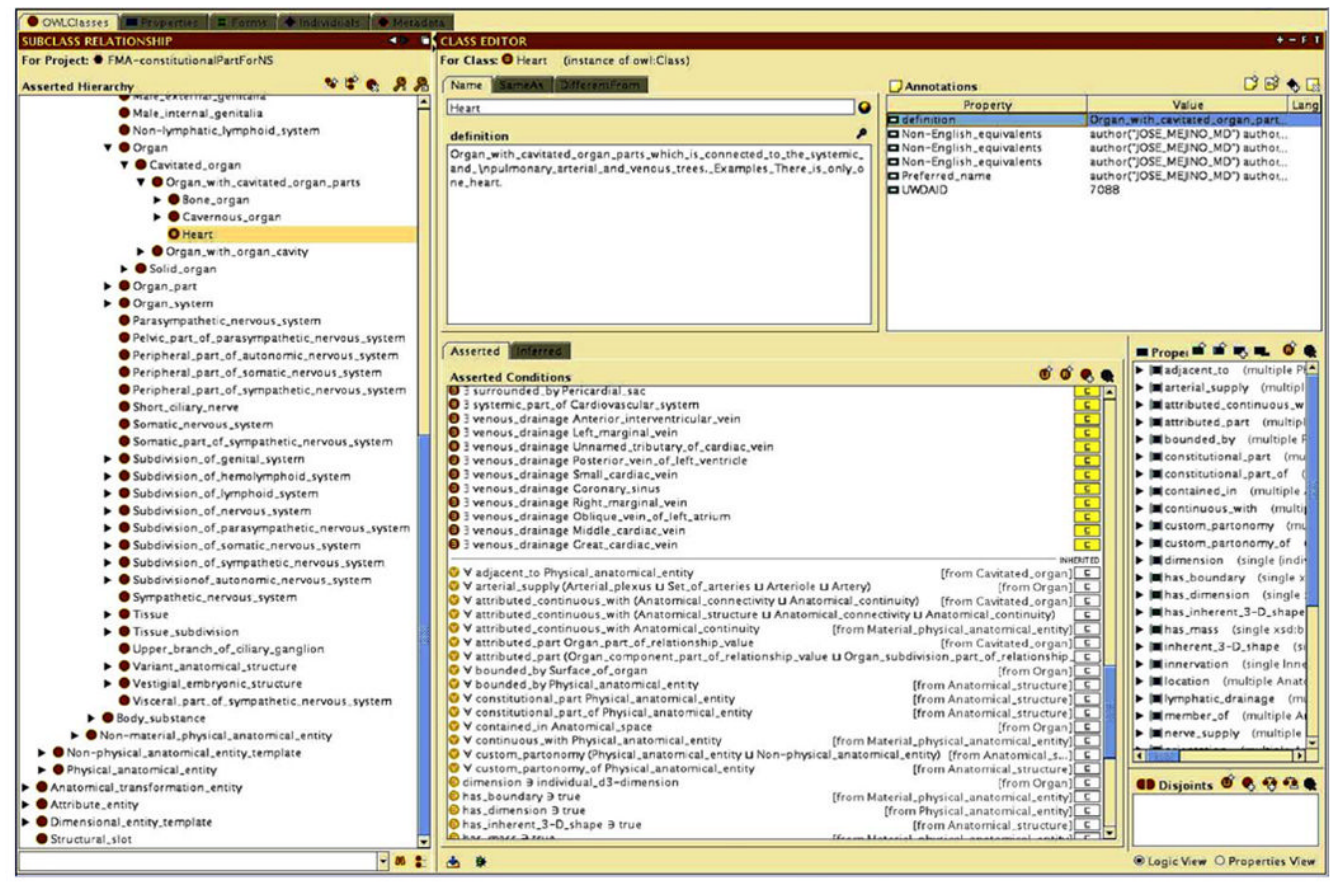

Fig. 3.

Class Heart and some of its asserted or inherited properties restrictions of the class Heart. 
Organ_with_cavitated_organ_parts

(3) $\exists$ constitutional_part Wall_of_heart

(3) $\exists$ constitutional_part Interatrial_septum

(3) $\exists$ constitutional_part Interventricular_septum

ق $\exists$ constitutional_part Atrioventricular_septum

(] $\exists$ constitutional_part Right_coronary_artery

(ᄏ $\exists$ constitutional_part Left_coronary_artery

] $\exists$ constitutional_part Coronary_sinus

] $\exists$ constitutional_part Great_cardiac_vein

ق $\exists$ constitutional_part Right_marginal_vein

ق $\exists$ constitutional_part Left_marginal_vein

(] $\exists$ constitutional_part Anterior_interventricular_vein

(3) $\exists$ constitutional_part Posterior_vein_of_left_ventricle

ق $\exists$ constitutional_part Middle_cardiac_vein

ㅋ $\exists$ constitutional_part Small_cardiac_vein

( $\exists$ constitutional_part Oblique_vein_of_left_atrium

Fig. 4.

The defined class Heart. 


\begin{tabular}{|c|c|}
\hline Heart in Protégé frames & Heart in OWL DL \\
\hline $\begin{array}{l}\text { Metaclass } \\
\begin{array}{l}\text { (defclass Heart } \\
\text { (is-a Organ_with_cavitated_organ_parts) } \\
\text {,... }\end{array}\end{array}$ & $\begin{array}{l}\text { <owl:Class rdf:ID="Heart"> } \\
\text { <owl:equivalentClass> } \\
\text { <owl:Class> } \\
\text { <owl:intersectionof rdf:parseType="Collection"> } \\
\text { <owl:Class rdf:about= } \\
\text { "\#Organ_with_cavitated_organ_parts"/> } \\
\text { <owl:Restriction> }\end{array}$ \\
\hline $\begin{array}{l}\text { Class (Instance of Metaclass) } \\
\text { ([Heart] } \\
\text { of organ_with_cavitated_organ_parts } \\
\text { (constitutional_part } \\
\text { Wall_of_heart } \\
\text { Cavity_of_left_atrium } \\
\text { Cavity_of_right_ventricle } \\
\text { Cavity_of_left_ventricle } \\
\text { Right_coronary_artery } \\
\text { Left_coronary_artery } \\
\text {... } \\
\text { Surface_of_heart) } \\
\text { (boundedby } \\
\text { (arterial_supply } \\
\text { Right_coronary_artery } \\
\text { Left_coronary_artery) } \\
\text {... }\end{array}$ & 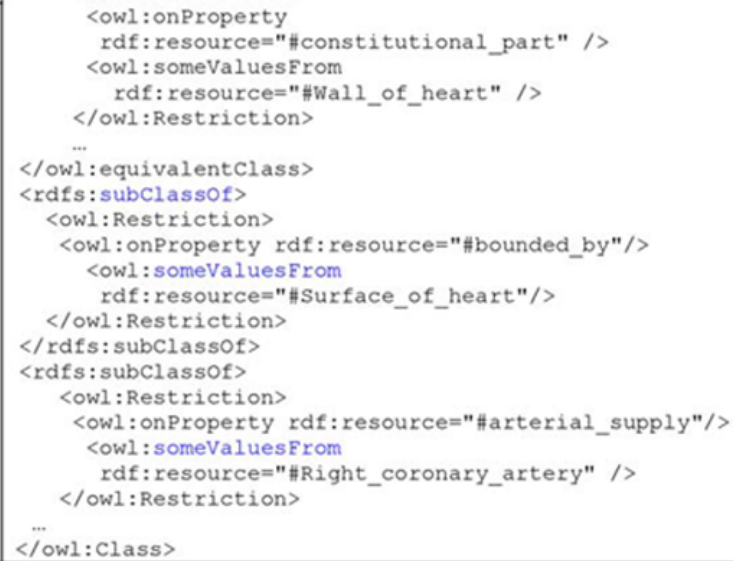 \\
\hline
\end{tabular}

Fig. 5.

Class Heart in OWL DL, derived from the Protégé metaclass and class definitions. 
Table 1

OWL DL constructors used for class description in the tests

\begin{tabular}{|c|c|c|c|c|c|c|c|c|c|c|c|c|c|c|c|}
\hline & \multicolumn{15}{|c|}{ Test } \\
\hline Test \# & 1 & 2 & 3 & 4 & 5 & 6 & 7 & 8 & 9 & 10 & 11 & 12 & 13 & 14 & 15 \\
\hline Success $(\mathrm{S})$ or Failure $(\mathrm{F})$ & $\mathbf{S}$ & $\mathbf{S}$ & $\mathbf{S}$ & $\mathbf{S}$ & $\mathbf{S}$ & $\mathbf{S}$ & $\mathbf{S}$ & $\mathbf{S}$ & $\mathbf{S}$ & $\mathbf{S}$ & $\mathbf{S}$ & $\mathbf{F}$ & $\mathbf{F}$ & $\mathbf{F}$ & $\mathbf{F}$ \\
\hline \multicolumn{16}{|l|}{ Classes Constructors } \\
\hline intersectionOf(C1 ...Cn) & & $\cdot$ & $\cdot$ & $\bullet$ & $\bullet$ & $\cdot$ & $\cdot$ & $\cdot$ & $\cdot$ & & & $\cdot$ & $\cdot$ & & \\
\hline unionOf(C1 ...Cn) & & $\cdot$ & $\cdot$ & $\cdot$ & - & $\cdot$ & - & $\cdot$ & $\cdot$ & - & - & $\cdot$ & - & $\bullet$ & $\bullet$ \\
\hline \multicolumn{16}{|l|}{ complementOf(C) } \\
\hline oneOf(o1... on $)$ & & & & & & & & & $\cdot$ & $\bullet$ & $\bullet$ & $\cdot$ & $\bullet$ & & \\
\hline \multicolumn{16}{|l|}{ Restriction R } \\
\hline allValuesFrom $(\mathrm{C})$ & & $\cdot$ & $\cdot$ & $\cdot$ & $\cdot$ & - & - & $\cdot$ & $\cdot$ & $\cdot$ & $\cdot$ & $\cdot$ & $\cdot$ & $\bullet$ & $\cdot$ \\
\hline someValuesFrom(C) & & - & - & $\cdot$ & - & - & - & - & - & - & - & - & - & - & - \\
\hline hasvalue(o) & & & & & & & & & $\cdot$ & & & - & $\bullet$ & & \\
\hline [minCardinality(n)] [maxCardinality(m)] [cardinality(l)] & & & & & & & & & & & & & & & \\
\hline \multicolumn{16}{|l|}{ Restriction T } \\
\hline \multicolumn{16}{|l|}{ allValuesFrom(D) someValuesFrom(D) } \\
\hline hasvalue (v) & & & & $\bullet$ & & & & & & & & & & & \\
\hline [minCardinality(n)] [maxCardinality(m)] [cardinality(l)] & & & & & & & & & & & & & & & \\
\hline \multicolumn{16}{|l|}{ Data Ranges } \\
\hline oneOf(v1 ...vn) & & & & & & & & & & & & & & & \\
\hline
\end{tabular}


Table 2

OWL DL Axioms and Facts used in the tests

\begin{tabular}{|c|c|c|c|c|c|c|c|c|c|c|c|c|c|c|c|}
\hline \multirow[b]{2}{*}{ Test \# } & \multicolumn{15}{|c|}{ Test } \\
\hline & 1 & 2 & 3 & 4 & 5 & 6 & 7 & 8 & 9 & 10 & 11 & 12 & 13 & 14 & 15 \\
\hline Success $(\mathrm{S})$ or Failure $(\mathrm{F})$ & $\mathbf{S}$ & $\mathbf{S}$ & $\mathbf{S}$ & $\mathbf{S}$ & $\mathbf{S}$ & $\mathbf{S}$ & $\mathbf{S}$ & $\mathbf{S}$ & $\mathbf{S}$ & $\mathbf{S}$ & $\mathbf{S}$ & $\mathbf{F}$ & $\mathbf{F}$ & $\mathbf{F}$ & $\mathbf{F}$ \\
\hline \multicolumn{16}{|l|}{ Class Axioms } \\
\hline \multicolumn{16}{|l|}{ EnumeratedClass $\left(A o_{1} \ldots o_{n}\right)$} \\
\hline \multicolumn{16}{|l|}{ DisjointClasses $\left(C_{1} \ldots C_{n}\right)$} \\
\hline EquivalentClasses $\left(C_{1} \ldots C_{n}\right)$ & & $\bullet$ & - & $\cdot$ & - & $\cdot$ & - & - & - & & & $\bullet$ & $\bullet$ & & \\
\hline SubClassOf $\left(\mathrm{C}_{1} \mathrm{C}_{2}\right)$ & & $\bullet$ & $\cdot$ & $\bullet$ & $\bullet$ & $\bullet$ & $\bullet$ & $\bullet$ & $\cdot$ & $\cdot$ & - & $\cdot$ & $\bullet$ & $\bullet$ & $\bullet$ \\
\hline \multicolumn{16}{|l|}{$\begin{array}{l}\text { Property Axioms } \\
\end{array}$} \\
\hline \multicolumn{16}{|l|}{ DatatypeProperty(T) } \\
\hline [Functional] & & & & $\bullet$ & & & & & & $\bullet$ & & $\bullet$ & $\bullet$ & & \\
\hline domain $(C)$ range(D) & & & & $\bullet$ & & & & & & $\bullet$ & & $\bullet$ & $\bullet$ & & \\
\hline \multicolumn{16}{|l|}{ EquivalentProperties $\left(T_{1} . . T_{n}\right)$ SubPropertyOf $\left(T_{1} T_{2}\right)$} \\
\hline \multicolumn{16}{|l|}{ ObjectProperty $(R)$} \\
\hline [inverseOf(R)] & & & & & & & & $\bullet$ & & & & $\bullet$ & $\bullet$ & $\bullet$ & \\
\hline [Functional] & & & & & & & & & $\cdot$ & & - & $\cdot$ & $\bullet$ & & \\
\hline \multicolumn{16}{|l|}{ [InverseFunctional] } \\
\hline [Symmetric] & & & & & & & & & & & & $\cdot$ & $\bullet$ & & $\bullet$ \\
\hline [Transitive] & & $\bullet$ & & & & & & & & & & & & & \\
\hline domain(C) range(D) & & $\bullet$ & $\cdot$ & $\bullet$ & $\bullet$ & $\bullet$ & $\bullet$ & $\bullet$ & $\cdot$ & $\cdot$ & • & $\cdot$ & $\cdot$ & $\bullet$ & $\bullet$ \\
\hline \multicolumn{16}{|l|}{ EquivalentProperties $\left(\mathrm{R}_{1} . . \mathrm{R}_{\mathrm{n}}\right)$ SubPropertyOf $\left(\mathrm{R}_{1} \mathrm{R}_{2}\right)$} \\
\hline \multicolumn{16}{|l|}{ Facts } \\
\hline Individual(o) & & & & $\bullet$ & & & & & $\cdot$ & & & $\cdot$ & $\cdot$ & & \\
\hline \multicolumn{16}{|l|}{ SameIndividual $\left(o_{1} \ldots o_{n}\right)$} \\
\hline DifferentIndividuals $\left(o_{1} \ldots o_{n}\right)$ & & & & $\cdot$ & & & & & $\bullet$ & & & $\cdot$ & $\bullet$ & & \\
\hline
\end{tabular}


Table A.1

Conversion of a non structural own slot into an Annotation Property

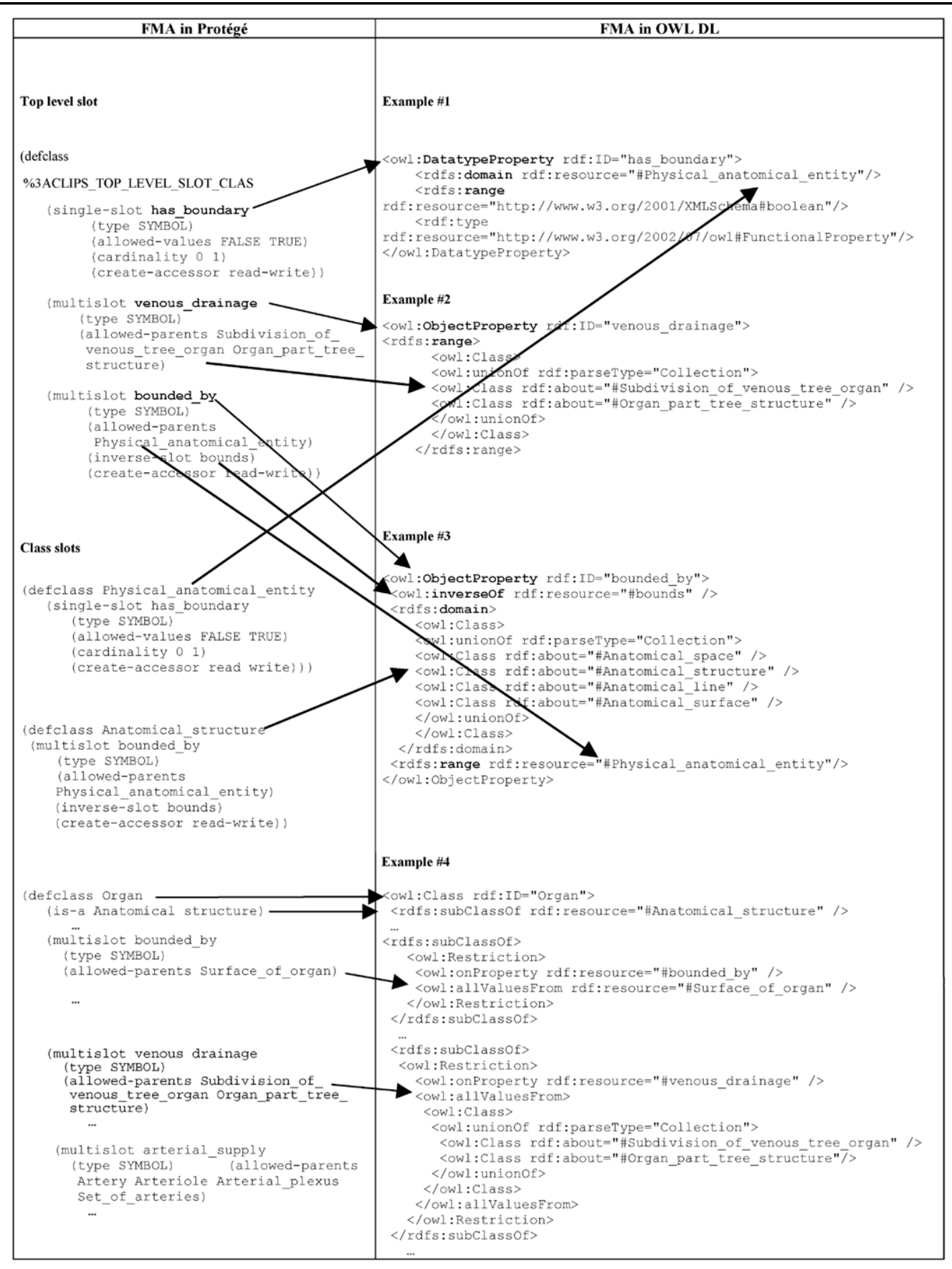

Web Semant. Author manuscript; available in PMC 2008 March 21. 
Table A.2

Conversion of a non structural own slot into an Annotation Property

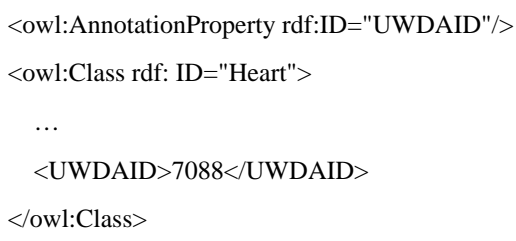




\section{Table A.3}

Conversion of a non structural own slot into an Annotation Property

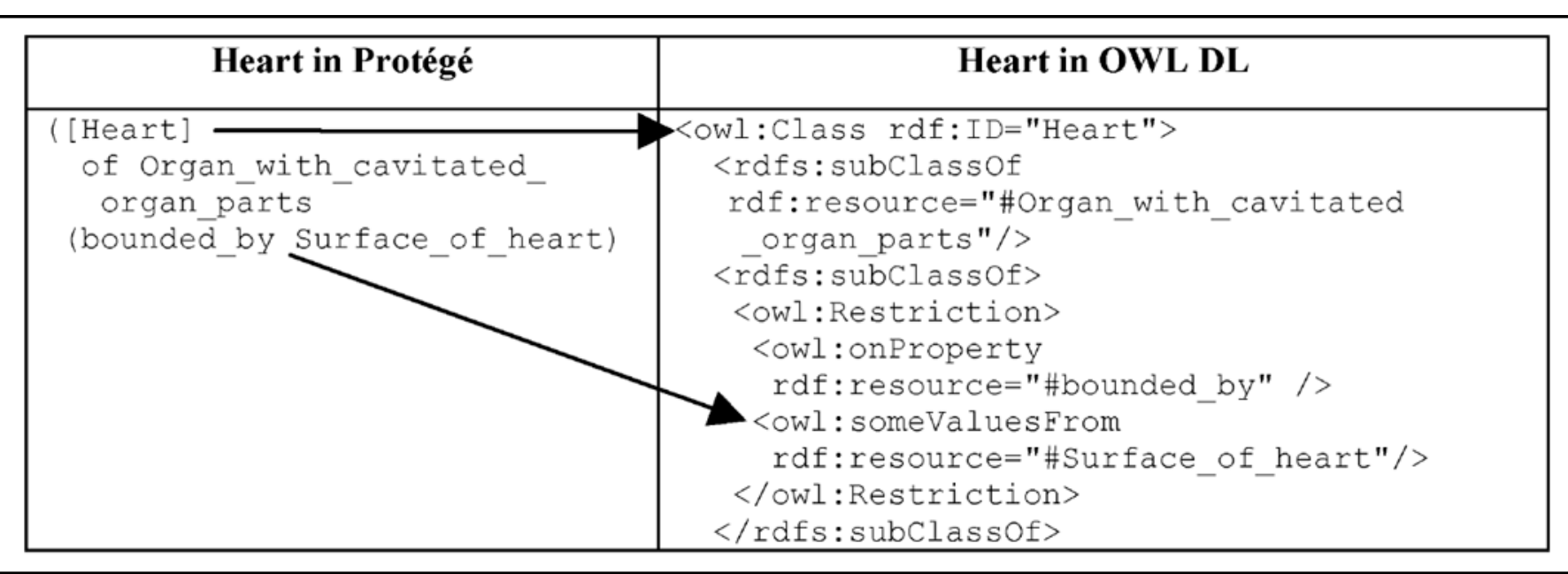


Table A.4

Conversion of a non structural own slot into an Annotation Property

\begin{tabular}{|c|c|}
\hline Heart in Protégé & Heart in OWL \\
\hline $\begin{array}{l}\text { ([Heart] } \\
\text { of Organ_with_- } \\
\text { cavitated_organ_parts } \\
\text { (attributed_part } \\
\text { [fm-live_12491] } \\
\text { [fm-live_12492] } \\
\text { [fm_live_17313] } \\
\text { [fm_live_17314] } \\
\text { [fm_live_17315] } \\
\text { [fm_live_17316] } \\
\text { [fm_live_17317] } \\
\text { [fm_live_17318] } \\
\text { [fm_live_17319] } \\
\text { [fm_live_17320] } \\
\text { [fm_live_17321] } \\
\text { [fm_live_17322] } \\
\text { [fm_live_17323]) } \\
\text { where } \\
\text { ([fm-live_12491] of Organ } \\
\text { subdivision_part_of_- } \\
\text { relationship_value } \\
\text { (anatomical_arbitrary } \\
\text { Arbitrary) } \\
\text { (partition Partition_2) } \\
\text { (related part Right_side } \\
\text { of_heart) } \\
\text { (shared_unshared } \\
\text { Unshared)) }\end{array}$ & 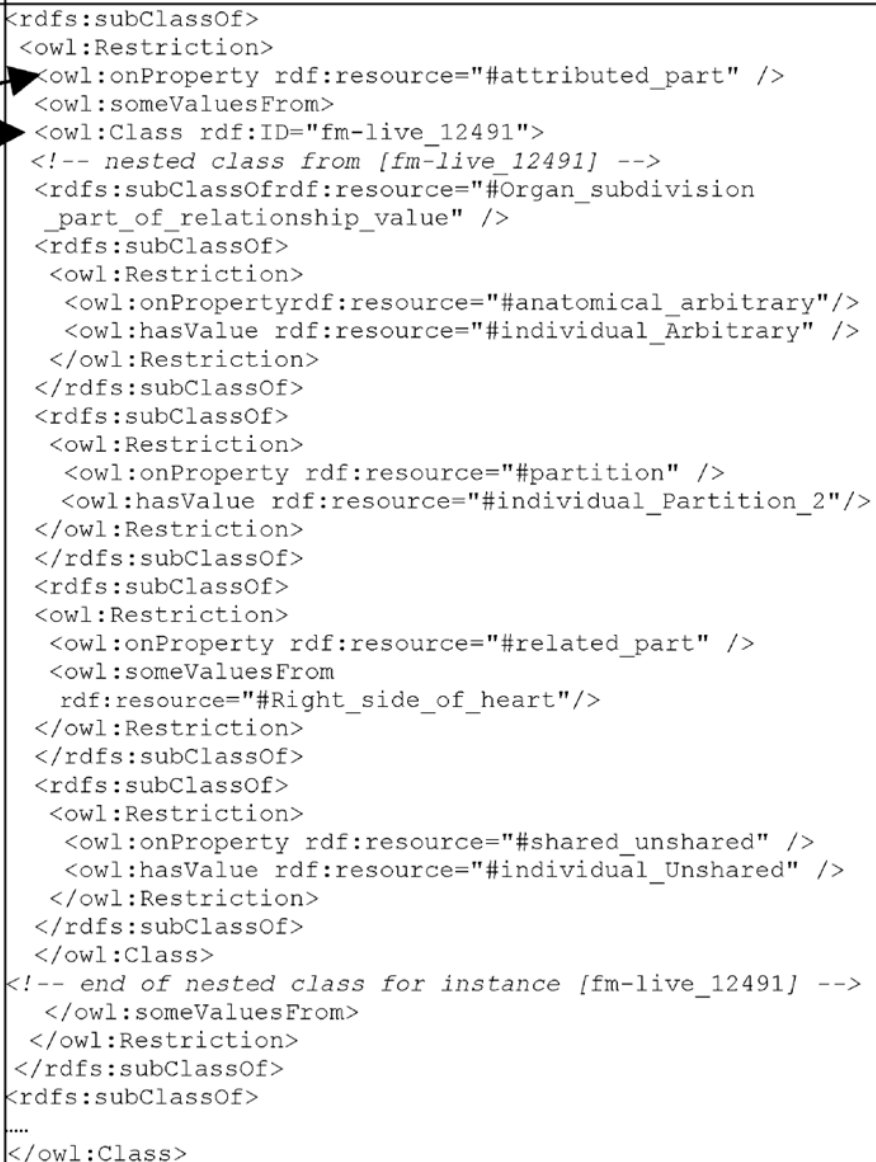 \\
\hline
\end{tabular}


Table A.5

Generating individuals in OWL DL

<owl:Thing rdf:ID=“individual_0-dimension" />
<owl:Thing rdf:ID=“individual_1-dimension" />
<owl:Thing rdf:ID=“individual_2-dimension" />
<owl:Thing rdf:ID=“"individual_3-dimension" /> 